\title{
AGO3 Gene
}

National Cancer Institute

\section{Source}

National Cancer Institute. AGO3 Gene. NCI Thesaurus. Code C84491.

This gene is involved in translational silencing. 